\title{
Full Counting Statistics for a Single-Electron Transistor, Non-equilibrium Effects at Intermediate Conductance
}

\author{
Yasuhiro Utsumi ${ }^{1,2}$, Dmitri S. Golubev ${ }^{1,3,4}$, and Gerd Schön ${ }^{1,4}$ \\ ${ }^{1}$ Institut für Theoretische Festköperphysik, Universität Karlsruhe, 76128 Karlsruhe, Germany \\ ${ }^{2}$ Condensed Matter Theory Laboratory, RIKEN, Wako, Saitama 351-0198, Japan \\ ${ }^{3}$ I.E. Tamm Department of Theoretical Physics, P.N. Lebedev Physics Institute, 119991 Moscow, Russia \\ ${ }^{4}$ Forschungszentrum Karlsruhe, Institut für Nanotechnologie, 76021 Karlsruhe, Germany
}

(Dated: February 2, 2008)

\begin{abstract}
We evaluate the current distribution for a single-electron transistor with intermediate strength tunnel conductance. Using the Schwinger-Keldysh approach and the drone (Majorana) fermion representation we account for the renormalization of system parameters. Nonequilibrium effects induce a lifetime broadening of the charge-state levels, which suppress large current fluctuations.
\end{abstract}

PACS numbers: $73.23 . \mathrm{Hk}, 72.70 .+\mathrm{m}$

The 'Full Counting Statistics' (FCS) of charge transport has proven to be a powerful tool in the description of current fluctuations [1]. The concept had been explored by Levitov and Lesovik [2], who expressed the FCS of an arbitrary mesoscopic structure with non-interacting electrons in terms of its $S$-matrix. Much less is known about the FCS of interacting mesoscopic systems, a problem which has been addressed only recently 3 , $, 4,5,6,6,7]$.

As a fundamental example of an interacting mesoscopic systems we consider a Single-Electron Transistor (SET). It consists of a metallic island coupled to drain and source (left and right) electrodes via low-capacitance tunnel junctions, with resistances $R_{\mathrm{L}}$ and $R_{\mathrm{R}}$, as well as to a gate electrode. The strength of Coulomb interaction is characterized by the charging energy $E_{C}=e^{2} / 2 C_{\Sigma}$, which depends on the total capacitance $C_{\Sigma}=C_{\mathrm{L}}+C_{\mathrm{R}}+C_{\mathrm{G}}$. A measure for the tunneling strength is the dimensionless parameter $\alpha_{0}=\left(R_{\mathrm{L}}+R_{\mathrm{R}}\right) / 2 \pi e^{2} R_{\mathrm{L}} R_{\mathrm{R}}$ (we put $\hbar=k_{\mathrm{B}}=1$ ).

In Refs. 3, 4] the FCS of a similar system - a quantum dot - has been studied, fully accounting for strong electron correlations, however only for a particular setup and parameters, corresponding to the Toulouse point. A renormalization group approach had been developed for the regime $\alpha_{0} \gg 1\left[\underline{5}\right.$. In the opposite limit, $\alpha_{0} \rightarrow 0$, the FCS has been analyzed to lowest order in Ref. [6] and next-to-lowest order (cotunneling) in Ref. 7]. However, effects of quantum fluctuations induced by nonvanishing $\alpha_{0}$ are still unknown. The aim of the present paper is to derive the FCS for a SET beyond perturbation theory in the intermediate strength tunneling regime $\alpha_{0} \lesssim 1$.

Let us further specify the situation to be considered. At low transport voltages and temperatures, $e V, T \ll E_{C}$, due to Coulomb blockade tunneling is suppressed in a SET, everywhere except near specific values of the gate voltage, e.g., near $Q_{\mathrm{G}} \equiv C_{\mathrm{G}} V_{\mathrm{G}}=e / 2$. In the neighborhood of this conductance peak the Coulomb barrier is $\Delta_{0}=E_{C}\left(1-2 Q_{\mathrm{G}} / e\right)$. For $\alpha_{0} \ll 1$ electrons tunnel via the island sequentially only when $\mu_{\mathrm{R}}<\Delta_{0}<\mu_{\mathrm{L}}$, where $\mu_{\mathrm{L} / \mathrm{R}}=\kappa_{\mathrm{L} / \mathrm{R}} e V$ is the voltage drop between the $\mathrm{L} / \mathrm{R}$ electrode and the island, and $\kappa_{\mathrm{L} / \mathrm{R}}= \pm C_{\mathrm{R} / \mathrm{L}}\left(C_{\mathrm{L}}+C_{\mathrm{R}}\right)^{-1}$.
With increasing $\alpha_{0}$, higher order effects such as cotunneling and quantum fluctuations of the charge gain importance [8]. They lead to a renormalization of $\Delta_{0}$ and $\alpha_{0}$. The perturbative renormalization group analysis [9] (for $\mathrm{eV}=0$ ) predicts a renormalization factor $z_{0}=1 /\left\{1+2 \alpha_{0} \ln \left(E_{C} / \Lambda\right)\right\}$ to depend logarithmically on the cutoff energy $\Lambda=\max \left\{\Delta_{0}, T\right\}$.

The model. - We concentrate on the tunneling regime with inverse $R C$ time $1 / R_{\mathrm{T}} C_{\Sigma}=4 \pi \alpha_{0} E_{C}$ smaller than $E_{C}$, which ensures that the charge-state levels are well resolved. In the vicinity of the conductance peak, precisely for $\left|\Delta_{0}\right| / E_{C} \ll 1$, it is sufficient to restrict attention to two charge states with charges differing by $e$. The Hamiltonian can then be mapped onto the 'multi-channel anisotropic Kondo model' [9]. Introducing a spin-1/2 operator $\hat{\sigma}$ acting on the charge states, we write

$$
\begin{aligned}
\hat{H}= & \sum_{\mathrm{r}=\mathrm{L}, \mathrm{R}, \mathrm{I}} \sum_{k n} \varepsilon_{\mathrm{r} k} \hat{a}_{\mathrm{r} k n}^{\dagger} \hat{a}_{\mathrm{r} k n}+\frac{\Delta_{0}}{2} \hat{\sigma}_{z} \\
& +\sum_{\mathrm{r}=\mathrm{L}, \mathrm{R}} \sum_{k k^{\prime} n}\left(T_{\mathrm{r}} \hat{a}_{\mathrm{I} k n}^{\dagger} \hat{a}_{\mathrm{r} k^{\prime} n} \hat{\sigma}_{+}+\text {H.c. }\right) .
\end{aligned}
$$

Here $\hat{a}_{\mathrm{rkn}}^{\dagger}$ creates an electron with wave vector $k$ and channel index (including spin) $n$ in the left or right electrode or island $(\mathrm{r}=\mathrm{L}, \mathrm{R}, \mathrm{I})$. Tunneling matrix elements $T_{\mathrm{r}}$ are assumed to be independent of $k$ and $n$. The junction conductances are $1 / R_{\mathrm{r}}=2 \pi e^{2} N_{\mathrm{ch}}\left|T_{\mathrm{r}}\right|^{2} \rho_{\mathrm{I}} \rho_{\mathrm{r}}$, with $N_{\mathrm{ch}}$ being the number of channels and $\rho_{\mathrm{r}}$ the electron DOS. We implicitly assume that energy and spin relaxation times are fast, and electrons obey the Fermi distribution.

A convenient tool to treat the spin- $1 / 2$ operators in Eq. (1) is the 'drone' (Majorana) fermion representation [1]], $\hat{\sigma}_{+}=\hat{c}^{\dagger} \hat{\phi}, \hat{\sigma}_{z}=2 \hat{c}^{\dagger} \hat{c}-1$. where $\hat{\phi}=\hat{d}^{\dagger}+\hat{d}$ is a Majorana fermion and $\hat{c}$ and $\hat{d}$ are Dirac fermions. This formulation enables one to apply Wick's theorem and the fermionic Schwinger-Keldysh approach [12, 13].

Cumulant generating functional. - The central object of our approach is the generating functional of connected Green's functions (GFs)

$$
W[\varphi] \equiv-i \ln \int D\left[a_{\mathrm{r} k n}^{*}, a_{\mathrm{r} k n}, c^{*}, c, d^{*}, d\right] e^{i \int_{C} d t \mathcal{L}(t)} .
$$


Here $\mathcal{L}$ is the Lagrangian corresponding to $\hat{H}$ (1), and six Grassmann variables have been introduced (see Ref. 14] for details). The closed time-path $C$ (Keldysh contour) runs from $t=-\infty$ to $\infty$, back to $-\infty$, and connects to the imaginary time-path to end at $t=-\infty-i / T$. We introduce auxiliary source fields, the phase of the tunneling matrix element, $T_{\mathrm{r}} \rightarrow T_{\mathrm{r}} e^{i \kappa_{\mathrm{r}} \varphi(t)}$, distinguishing between forward and backward time-paths, $\varphi_{+}(t)$ and $\varphi_{-}(t)$. The 'center-of-mass' variable $\varphi_{c}(t) \equiv\left\{\varphi_{+}(t)+\right.$ $\left.\varphi_{-}(t)\right\} / 2=e V t$ is then fixed by the transport voltage.

From the Cumulant Generating Functional (CGF) one finds the number of transmitted electrons $q$ during the measurement time $t_{0}, \mathcal{W}(\lambda)=\sum_{n=0}^{\infty}\left\langle\left\langle\delta q^{n}\right\rangle\right\rangle(i \lambda)^{n} / n$ !. Following Ref. [13], it is derived from Eq. (2) by fixing during the measurement the 'counting field' (relative variable) $\varphi_{\Delta}(t) \equiv \varphi_{+}(t)-\varphi_{-}(t)$ at a constant value $\lambda$ :

$$
\mathcal{W}(\lambda)=\left.i W[\varphi]\right|_{\varphi_{c}(t)=e V t, \varphi_{\Delta}(t)=\lambda \theta\left(t_{0} / 2+t\right) \theta\left(t_{0} / 2-t\right)} .
$$

The distribution of $q$ (or equivalently of the current $I \equiv$ $\left.e q / t_{0}\right)$ is given by the inverse Fourier transformation,

$$
P=\frac{1}{2 \pi} \int_{-\pi}^{\pi} d \lambda e^{\mathcal{W}(\lambda)-i q \lambda} \approx e^{\mathcal{W}\left(\lambda^{*}\right)-i\left(t_{0} I / e\right) \lambda^{*}} .
$$

The integral can be evaluated in saddle point approximation, with $\lambda^{*}$ following from the relation $I=$ $-i e \partial_{\lambda} \mathcal{W}\left(\lambda^{*}\right) / t_{0}[\underline{6}$. This approach is valid for long measurement times since $\mathcal{W}$ is proportional to $t_{0}$ (see below).

We proceed following Ref. 14], where a conserving approximation for the second cumulant had been developed. Tracing out the electron degrees of freedom leads to an effective action for the $c$ and $d$ fields, $S^{\lambda} \equiv S_{\mathrm{ch}}+S_{t}^{\lambda}$, composed of a charging and a tunneling term:

$$
\begin{aligned}
& S_{\mathrm{ch}}=\int_{C} d t\left\{c(t)^{*}\left(i \partial_{t}-\Delta_{0}\right) c(t)+i d(t)^{*} \partial_{t} d(t)\right\}, \\
& S_{t}^{\lambda}=-\int_{C} d t d t^{\prime} c^{*}(t) \phi(t) \alpha^{\lambda}\left(t, t^{\prime}\right) \phi\left(t^{\prime}\right) c\left(t^{\prime}\right)+O\left(T_{\mathrm{r}}^{4}\right) .
\end{aligned}
$$

Here $\alpha^{\lambda}=\alpha_{\mathrm{L}}^{\lambda}+\alpha_{\mathrm{R}}^{\lambda}$ is a particle-hole GF describing tunneling of an electron from one electrode to the island. It depends on the counting field. The connection to the ordinary GF is established by a rotation by $\lambda_{\mathrm{r}}=\kappa_{\mathrm{r}} \lambda$ in the Keldysh space as follows:

$$
\tilde{\alpha}_{\mathrm{r}}^{\lambda}(\omega)=U_{\lambda_{\mathrm{r}}}^{\dagger} \tilde{\alpha}_{\mathrm{r}}(\omega) U_{\lambda_{\mathrm{r}}}, \tilde{\alpha}_{\mathrm{r}}(\omega)=\left(\begin{array}{cc}
0 & \alpha_{\mathrm{r}}^{A}(\omega) \\
\alpha_{\mathrm{r}}^{R}(\omega) & \alpha_{\mathrm{r}}^{K}(\omega)
\end{array}\right),
$$

where $U_{\lambda_{\mathrm{r}}}=\exp \left(-i \lambda_{\mathrm{r}} \boldsymbol{\tau}_{1} / 2\right)$ and $\left[\boldsymbol{\tau}_{1}\right]_{i j}=1-\delta_{i j}$. The retarded and advanced components are given by

$$
\alpha_{\mathrm{r}}^{R}(\omega)=\alpha_{\mathrm{r}}^{A}(\omega)^{*}=-i \pi \alpha_{0}^{\mathrm{r}} \frac{\left(\omega-\mu_{\mathrm{r}}\right) E_{C}^{2}}{\left(\omega-\mu_{\mathrm{r}}\right)^{2}+E_{C}^{2}},
$$

where $\alpha_{0}^{\mathrm{r}}=1 / 2 \pi e^{2} R_{\mathrm{r}}$, and the Keldysh component by $\alpha_{\mathrm{r}}^{K}(\omega)=2 \alpha_{\mathrm{r}}^{R}(\omega) \operatorname{coth}\left(\omega-\mu_{\mathrm{r}}\right) / 2 T$. We introduced a Lorentzian cutoff to regularize the ultraviolet divergence and ignored the term $O\left(T_{\mathrm{r}}^{4}\right)$ in the action (6), since it is small in the limit $N_{\mathrm{ch}} \gg 1$.

The free retarded GF of the Dirac fermion $\hat{c}, g_{c}^{R}(\omega)=$ $1 /\left(\omega+i \eta-\Delta_{0}\right)$, describes the dynamics of charge excitations ( $\eta$ is a positive infinitesimal). The corresponding self-energy $\Sigma_{c}^{\lambda}=\Sigma_{\mathrm{L}}^{\lambda}+\Sigma_{\mathrm{R}}^{\lambda}$ accounts for quantum fluctuations of the island charge caused by tunneling. Integrating out $d$-fields, we obtain the components of the self-energy in first order in $\alpha_{0}$ :

$$
\Sigma_{\mathrm{r}}^{K}(\omega)=2 \alpha_{\mathrm{r}}^{R}(\omega), \quad \Sigma_{\mathrm{r}}^{R}(\omega)=\int \frac{d \omega^{\prime}}{2 \pi} \frac{i \alpha_{\mathrm{r}}^{K}\left(\omega^{\prime}\right)}{\omega+i \eta-\omega^{\prime}} .
$$

(For simplicity we present here only the result for $\lambda=0$.) For a symmetric SET $\left(R_{\mathrm{L}}=R_{\mathrm{R}}, C_{\mathrm{L}}=C_{\mathrm{R}}\right)$, at $T=0$ and $|\omega| \ll e V$, one finds $\Sigma_{c}^{R}(\omega) \approx \alpha_{0} \ln \left(2 E_{C} / e V\right) \omega-i \Gamma / 2$, where $\Gamma=\Gamma_{\mathrm{IL}}+\Gamma_{\mathrm{LI}}+\Gamma_{\mathrm{IR}}+\Gamma_{\mathrm{RI}}$ is the sum of the rates $\Gamma_{\mathrm{rI} / \mathrm{Ir}}= \pm\left(1 / e^{2} R_{\mathrm{r}}\right)\left(\Delta_{0}-\mu_{\mathrm{r}}\right) /\left(e^{ \pm\left(\Delta_{0}-\mu_{\mathrm{r}}\right) / T}-1\right)$ describing tunneling into (out of) the island through the junction $\mathrm{r}$, evaluated by Fermi's golden rule.

We can proceed in a systematic diagrammatic expansion in $\alpha_{0}$ [14]. To lowest order one obtains for the $\mathrm{CGF}: \mathcal{W}^{[1]}(\lambda)=-\int_{C} d t d t^{\prime} g_{c}\left(t, t^{\prime}\right) \Sigma_{c}^{\lambda}\left(t^{\prime}, t\right)$. We project the time from the Keldysh contour $C$ to the real axis and observe that for long enough measurement times we can approximate $\delta_{t_{0}}(\omega) \equiv \int_{-t_{0} / 2}^{t_{0} / 2} d t e^{-i \omega t} / 2 \pi$ by a $\delta$-function, $\delta_{t_{0}}(\omega) \rightarrow \delta(\omega)$, and $\left(\delta_{t_{0}}(\omega)\right)^{2} \rightarrow t_{0} \delta(\omega) / 2 \pi$. The latter ensures that any closed diagram, consequently $\mathcal{W}$, is proportional to $t_{0}$. After Fourier transformation we obtain

$$
\begin{aligned}
& \mathcal{W}^{[1]}(\lambda) \approx-t_{0} \int d \omega \operatorname{Tr}\left\{\tilde{g}_{c}(\omega) \boldsymbol{\tau}_{1} \tilde{\Sigma}_{c}^{\lambda}(\omega) \boldsymbol{\tau}_{1}\right\} / 2 \pi \\
& =t_{0} \sum_{\mathrm{r}=\mathrm{L}, \mathrm{R}}\left\{P_{-} \Gamma_{\mathrm{rI}}\left(e^{i \lambda_{\mathrm{r}}}-1\right)+P_{+} \Gamma_{\mathrm{Ir}}\left(e^{-i \lambda_{\mathrm{r}}}-1\right)\right\} .
\end{aligned}
$$

Here we used the expression for the Keldysh component of $c$-field GF, $g_{c}^{K}(\omega)=2 i \operatorname{Im} g_{c}^{R}(\omega)\left(P_{-}-P_{+}\right)$, which contains equilibrium occupation probabilities of the charge states $Q_{\mathrm{G}}$ and $Q_{\mathrm{G}}-e: P_{ \pm}=1 /\left(e^{ \pm \Delta_{0} / T}+1\right)$.

At this point we note that the naive first order expansion (2) is insufficient. First, it contains the equilibrium occupation probabilities rather than the stationary ones. Second, due to charge conservation the CGF should depend only on the difference of the counting fields $\lambda_{\mathrm{L}}-\lambda_{\mathrm{R}}=\lambda[15$, which is also violated. These problems are resolved if we sum up an infinite subclass of diagrams. Specifically, we sum up the geometric series in $\left(\tilde{g}_{c} \boldsymbol{\tau}_{1} \tilde{\Sigma}_{c}^{\lambda} \boldsymbol{\tau}_{1}\right)$, which contains the leading logarithms, i.e. powers of $\alpha_{0} \ln \left(2 E_{C} / \mathrm{eV}\right)$, and get

$$
\begin{aligned}
& \mathcal{W}(\lambda)=t_{0} \int \frac{d \omega}{2 \pi} \operatorname{Tr} \ln \left[\tilde{g}_{c}(\omega)^{-1}-\boldsymbol{\tau}_{1} \tilde{\Sigma}_{c}^{\lambda}(\omega) \boldsymbol{\tau}_{1}\right] \\
& =t_{0} \int \frac{d \omega}{2 \pi} \ln \left[1+T^{F}(\omega) f\left(\omega-\mu_{\mathrm{L}}\right) h\left(\omega-\mu_{\mathrm{R}}\right)\left(e^{i \lambda}-1\right)\right. \\
& \left.+T^{F}(\omega) f\left(\omega-\mu_{\mathrm{R}}\right) h\left(\omega-\mu_{\mathrm{L}}\right)\left(e^{-i \lambda}-1\right)\right] .
\end{aligned}
$$


Here $f(\omega)=1 /\left(e^{\omega / T}+1\right), h(\omega)=1-f(\omega)$ and

$$
T^{F}(\omega)=-\alpha_{\mathrm{L}}^{K}(\omega) \alpha_{\mathrm{R}}^{K}(\omega) /\left|\omega-\Delta_{0}-\Sigma_{c}^{R}(\omega)\right|^{2} .
$$

Note that we subtracted a constant from the CGF in order to satisfy the normalization condition $\mathcal{W}(0)=0$.

Eq. (10) is the main result of this paper. It is similar to Levitov-Lesovik formula [2], but the effective transmission probability (11) accounts for quantum fluctuations of the charge. Using the condition Eq. (3), Eq. (10) can also be obtained from an approximate $W$ given in Eq. (25) of Ref. 14]. Thus, the first and second cumulants, $\langle I\rangle=e\langle\langle\delta q\rangle\rangle / t_{0}$ and $S_{I I}=2 e^{2}\left\langle\left\langle\delta q^{2}\right\rangle\right\rangle / t_{0}$, reproduce the average current [10] and zero-frequency noise [14], derived before.

Although we used only the first order expansion for the self-energy, Eq. (10) is exact to second order in $\alpha_{0}$. One can check that the diagrams ignored in Eq. (10) within second order expansion, i.e. the diagrams with intersecting interaction lines, are proportional to $\Delta_{0} / E_{C} \ll 1$. Furthermore, higher order terms of Eq. (10) generate the renormalization factor $z_{0}$ consistent with the renormalization group result [9].

Limiting cases. - In the limit $\alpha_{0} \rightarrow 0$, Eq. (10) reproduces the result of the 'orthodox' theory [6]:

$$
\begin{aligned}
& \mathcal{W}^{(1)}(\lambda)=t_{0} \Gamma \frac{\sqrt{D(\lambda)}-1}{2}, \\
& D(\lambda)=1+\frac{4 \Gamma_{\mathrm{LI}} \Gamma_{\mathrm{IR}}\left(e^{i \lambda}-1\right)}{\Gamma^{2}}+\frac{4 \Gamma_{\mathrm{RI}} \Gamma_{\mathrm{IL}}\left(e^{-i \lambda}-1\right)}{\Gamma^{2}} .
\end{aligned}
$$

The second order expansion in $\alpha_{0}$ reads,

$$
\mathcal{W}^{(2)}(\lambda)=\partial_{\Delta_{0}}\left\{\operatorname{Re} \Sigma_{c}^{R}\left(\Delta_{0}\right) \mathcal{W}^{(1)}(\lambda)\right\}+\mathcal{W}^{\cot }(\lambda) .
$$

The first term of this equation provides the renormalization of the system parameters up to first order in $\alpha_{0}$. Namely, the parameters are renormalized as $\alpha_{0} \rightarrow$ $\alpha_{0}\left\{1+\partial_{\Delta_{0}} \operatorname{Re} \Sigma_{c}^{R}\left(\Delta_{0}\right)\right\}$ and $\Delta_{0} \rightarrow \Delta_{0}+\operatorname{Re} \Sigma_{c}^{R}\left(\Delta_{0}\right)$. This agrees with the corresponding results obtained earlier for the average current [16]. It is also consistent with the recent results of Braggio et al. 7]. We also checked that Eq. (14) can be reproduced by the systematic real-time diagrammatic expansion similar to that of Ref. [10].

The second term of Eq. (14) is the CGF of a bidirectional Poissonian process

$$
\mathcal{W}^{\cot }(\lambda)=t_{0}\left\{\gamma^{+}\left(e^{i \lambda}-1\right)+\gamma^{-}\left(e^{-i \lambda}-1\right)\right\},
$$

governed by the cotunneling rates

$\gamma^{ \pm}=\int d \omega \frac{2 \pi \alpha_{0}^{\mathrm{L}} \alpha_{0}^{\mathrm{R}}\left(\omega-\mu_{\mathrm{L}}\right)\left(\omega-\mu_{\mathrm{R}}\right)}{\left(e^{ \pm \frac{\omega-\mu_{\mathrm{L}}}{T}}-1\right)\left(1-e^{\mp \frac{\omega-\mu_{\mathrm{R}}}{T}}\right)} \operatorname{Re} \frac{1}{\left(\omega+i \eta-\Delta_{0}\right)^{2}}$.

This term is relevant in the Coulomb blockade regime and is consistent with the FCS theory of quasiparticle tunneling [17].
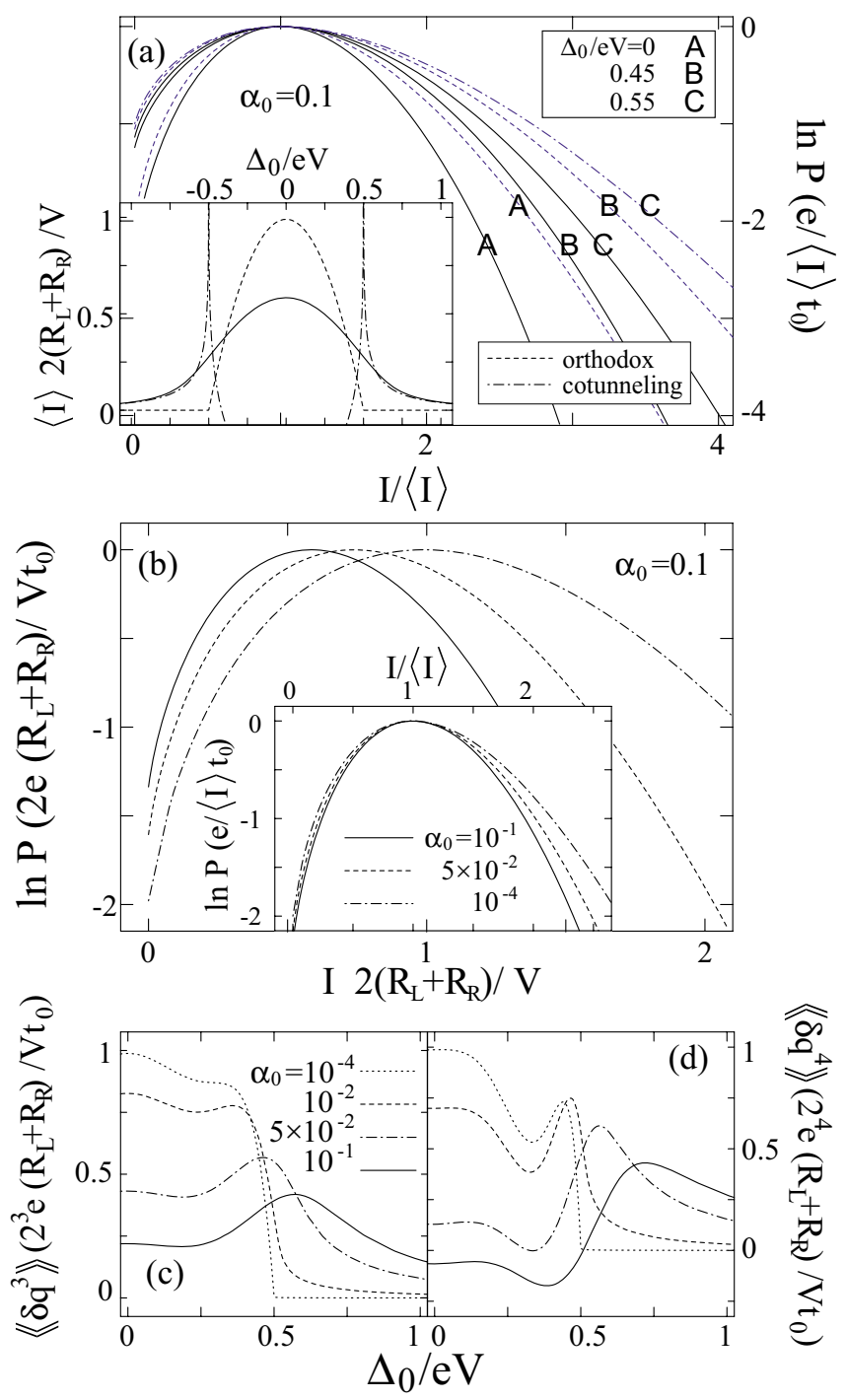

FIG. 1: Current distribution $P$ of a symmetric SET $[T=0$ and $\left.e V / E_{C}=0.2\right]$. (a) Solid lines are plots of $P$ for various values of $\Delta_{0}$. The dashed lines represent the 'orthodox' theory and the dot-dashed line represents the cotunneling expansion. The inset shows the average current for the same parameters. (b) Plot of $P$ at $\Delta_{0}=0$ for various values of the conductance as a function of the current normalized to $V / 2\left(R_{\mathrm{L}}+R_{\mathrm{R}}\right)$; inset: the same distribution normalized to the average current $\langle I\rangle$. (c) The skewness and (d) kurtosis for various conductances.

At the conductance peak, $\Delta_{0}=0$, for a symmetric SET and $T=0$, the 'orthodox' theory yields $\mathcal{W}^{(1)} \approx$ $2 \bar{q}\left(e^{i \lambda / 2}-1\right)$, where $e \bar{q} / t_{0}=V / 2\left(R_{\mathrm{L}}+R_{\mathrm{R}}\right)$. The factor $e^{i \lambda / 2}$ leads to a sub-Poissonian value of the Fano factor $S_{I I} / 2 e\langle I\rangle \approx 1 / 2$, indicating that tunneling processes are correlated. The origin of this correlation can be understood from the explicit form of the distribution $P(q)=\sum_{q_{\mathrm{L}}, q_{\mathrm{R}}=0}^{\infty} P_{\mathrm{P}}\left(q_{\mathrm{L}}\right) P_{\mathrm{P}}\left(q_{\mathrm{R}}\right) \delta_{q,\left(q_{\mathrm{L}}+q_{\mathrm{R}}\right) / 2}$, obtained by inverse Fourier transformation of Eq. (4) without saddle point approximation. The numbers of electrons transmitted through either junction, $q_{\mathrm{L}}$ and $q_{\mathrm{R}}$, follow the 
same Poissonian distribution $P_{\mathrm{P}}(q)=\bar{q}^{q} e^{-\bar{q}} / q$ !. The Kronecker delta implies that $q_{\mathrm{L}}$ and $q_{\mathrm{R}}$ are correlated.

With $\Delta_{0}$ approaching the threshold value $\Delta_{0} / \mathrm{eV}=$ 0.5 , the tunneling onto the island becomes the bottleneck and the CGF acquires the Poissonian form, $\mathcal{W}^{(1)} \approx$ $t_{0} \Gamma_{\mathrm{LI}}\left(e^{i \lambda}-1\right)$. It remains Poissonian in the cotunneling regime, $\left|\Delta_{0} / e V\right|>0.5, \mathcal{W}^{\text {cot }} \approx t_{0} \gamma^{+}\left(e^{i \lambda}-1\right)$.

Let us compare our result (10) to the 'orthodox' and the cotunneling theories. The latter theories fail around the threshold $\Delta_{0} / \mathrm{eV}=0.5$. For example, in the average current, shown in the inset of Fig. 1(a), we observe a mismatch between their predictions since $\Gamma_{\mathrm{LI}} \rightarrow 0$ while $\gamma^{+} \rightarrow \infty$. In contrast, the distribution derived from Eq. (10), shown in Fig. 1(a) by solid lines, behaves regular. It widens with increasing $\Delta_{0}$. The 'orthodox' (dashed lines) and the cotunneling (dot-dashed line) theories show the same trend but overestimate the width.

Renormalization and lifetime broadening effects. - For large conductance, quantum fluctuations of the charge are pronounced. However, as long as $z_{0} \Gamma \ll \Lambda$, where $\Lambda=\max \left(\left|z_{0} \Delta_{0}\right|, 2 \pi T,|e V| / 2\right)$, the 'orthodox' CGF $\mathcal{W}^{(1)}$ with renormalized parameters $z_{0} \alpha_{0}$ and $z_{0} \Delta_{0}$ remains a good approximation. This scenario fails in the regime $\Lambda \ll T_{K}=E_{C} e^{-1 / 2 \alpha_{0}} / 2 \pi$ where the approximation of leading logarithms becomes insufficient.

The renormalization effect is illustrated in Fig. 1(b), where the current distribution at $\Delta_{0}=0$ is plotted. Since $z_{0}$ decreases with increasing $\alpha_{0}$, the mean value of the current, i.e. a peak position, shifts to lower values. The renormalization effect can be absorbed when we plot $\ln P$ with horizontal axis normalized by $\langle I\rangle$ rather than $V / 2\left(R_{\mathrm{L}}+R_{\mathrm{R}}\right)$. However, even after plotting the distribution as a function of the normalized current [inset of Fig. [(b)] the three curves do not collapse to a single one. The remaining differences can be attributed to the non-Markovian lifetime broadening effect as described by $\operatorname{Im} \Sigma_{c}^{R}$. We observe that the current distribution shrinks with increasing $\alpha_{0}$. This agrees with the previously noted suppression of the Fano factor [14]. FCS provides further information, showing in detail how the probability for currents exceeding the average value is suppressed.

The effect of lifetime broadening is also visible in the moments. At moderately large voltages, $e V \gg T_{\mathrm{K}}$, and at $T=0$ the real part of the self-energy $\Sigma_{c}^{R}$ is negligible and $\Sigma_{c}^{R}(\omega) \approx-i \pi \alpha_{0} e V$. The CGF at $\Delta_{0}=0$ then is

$$
\begin{aligned}
\mathcal{W}(\lambda) & \approx 2 \bar{q}\left\{\left(e^{i \lambda / 2}-1\right)-2 \alpha_{0}\left(e^{i \lambda}-1\right)\right. \\
& \left.+\pi^{2} \alpha_{0}^{2}\left(e^{i 3 \lambda / 2}-e^{i \lambda / 2}\right) / 2+O\left(\alpha_{0}^{3}\right)\right\},
\end{aligned}
$$

and the ratio of higher order cumulants to the first one becomes $\left\langle\left\langle\delta q^{n}\right\rangle\right\rangle /\langle\langle\delta q\rangle\rangle=2^{1-n}\left\{1-4 \alpha_{0}\left(2^{n-1}-1\right)+\right.$ $\left.O\left(\alpha_{0}^{2}\right)\right\}$. We note that, as $\alpha_{0}$ increases, higher order cumulants are suppressed as compared to the Poissonian result $2^{1-n}$.

Figures 1(c) and (d) show the skewness $\left\langle\left\langle\delta q^{3}\right\rangle\right\rangle$ and the kurtosis $\left\langle\left\langle\delta q^{4}\right\rangle\right\rangle$ as a function of $\Delta_{0}$. A peak around the threshold develops with increasing conductance. We expect that this kind of behavior can be observed with present-day experimental techniques [18].

In conclusion, we have derived the Full Counting Statistics for a single-electron transistor in the vicinity of a conductance peak. Quantum fluctuations of the charge are taken into account by a summation of a certain subclass of diagrams, which corresponds to the leading logarithmic approximation. In first order in $\alpha_{0}$ our results reproduce the 'orthodox' theory, while in second order they account for renormalization and cotunneling effects. We have shown that in non-equilibrium situations quantum fluctuations of the charge induce lifetime broadening for the charge states of the central island. An important consequence is the suppression of the probability for currents larger than the average value.

We thank D. Bagrets, A. Braggio, Y. Gefen, J. König, A. Shnirman for valuable discussions. YU was supported by the DFG "Center for Functional Nanostructures" and RIKEN Special Postdoctoral Research Program.

[1] Quantum Noise in Mesoscopic Physics, NATO Science Series II, Vol. 97, ed. Yu.V. Nazarov (Kluwer, 2003).

[2] L.S. Levitov and G.B. Lesovik, JETP lett. 58, 230 (1993); cond-mat/9401004.

[3] M. Kindermann and B. Trauzettel, Phys. Rev. Lett. 94, 166803 (2005).

[4] A. Komnik and A.O. Gogolin, Phys. Rev. Lett. 94, 216601 (2005).

[5] D.A. Bagrets and Yu.V. Nazarov, Phys. Rev. Lett. 94, 056801 (2005).

[6] D.A. Bagrets and Yu.V. Nazarov, Phys. Rev. B 67, 085316 (2003).

[7] A. Braggio, J. König, and R. Fazio, cond-mat/0507527

[8] D.S. Golubev et al., Phys. Rev. B 56, 15782 (1997), and further references therein.

[9] K.A. Matveev, Sov. Phys. JETP. 72, 892 (1991).

[10] H. Schoeller and G. Schön, Phys. Rev. B 50, 18436 (1994).

[11] H.J. Spencer and S. Doniach, Phys. Rev. Lett. 18, 994 (1967).

[12] K.-C. Chou, Z.-B. Su, B.-L. Hao, and L. Yu, Phys. Rep. 118, 1 (1985); A. Kamenev, cond-mat/0412296

[13] A. Kamenev in Strongly Correlated Fermions and Bosons in Low-Dimensional Disordered Systems, eds. I. V. Lerner et al., NATO Science Ser. II, Vol. 72 (Kluwer, Dordrecht, 2002).

[14] Y. Utsumi et al., Phys. Rev. B 67, 035317 (2003).

[15] W. Belzig in CFN Lecture Notes on Functional Nanostructures Vol. 1, eds. K. Busch et al. Lecture Notes in Physics, Vol. 658 (Springer-Verlag, Berlin, 2004).

[16] J. König, H. Schoeller, and G. Schön, Phys. Rev. Lett. 78, 4482 (1997); Phys. Rev. B 58, 7882 (1998).

[17] L.S. Levitov and M. Reznikov, Phys. Rev. B 70, 115305 (2004).

[18] B. Reulet et al., Phys. Rev. Lett. 91, 196601 (2003). 